\title{
ANTARES offline study of three alerts after Baikal-GVD follow-up found coincident cascade neutrino events
}

\section{S. Alves Garre ${ }^{a, *}$ and F. Versari ${ }^{b, c}$ on behalf of the ANTARES Collaboration}

\section{A.D. Avrorin $^{d}$, Zhan-Arys M. Dzhlkibaev $^{d}$, M.D. Shelepov ${ }^{d}$ and O.V. Suvorova ${ }^{d}$ on} behalf of the Baikal-GVD Collaboration

(a complete list of authors can be found at the end of the proceedings)

${ }^{a}$ Instituto de Física Corpuscular, UV-CSIC,

Calle Catedrático José Beltrán, $n^{\circ} 2$ E-46980, Paterna, Spain

${ }^{b}$ INFN-Bologna

Viale Berti Pichat, 6/2, 40127, Bologna, Italy

${ }^{c}$ Université de Paris, CNRS, Astroparticule et Cosmologie,

F-75013 Paris, France

${ }^{d}$ Institute for Nuclear Research, RAS,

prospekt 60-letiya Oktyabrya 7a, Moscow 117312

E-mail: Sergio.Alves@ific.uv.es, federico.versari@bo.infn.it,

djilkib@yandex.ru, shelepov-1992@mail.ru, suvorova@inr.ru

ANTARES and Baikal-GVD are both Cherenkov neutrino telescopes located in the Northern Hemisphere so their fields of view almost overlap allowing for a combined study of the sky. ANTARES sends alerts after a fast online analysis based on energy and reconstruction direction of track-like events. From December of 2018 until the beginning of 2021, Baikal-GVD received 38 ANTARES alerts, and followed up 32. No coincidence was found. However, a search of the Baikal-GVD cascade sample showed some events falling within an angular distance of less than $5^{\circ}$ for three of the ANTARES alerts in a time span of 48 hours. A dedicated offline analysis based on the full ANTARES data sample has been started to search for additional coincident tracks and cascades at a $3 \sigma$ significance. In this work we present the final results of the offline analysis of the three ANTARES alerts: limits on the astrophysical neutrino fluence are reported.

$37^{\text {th }}$ International Cosmic Ray Conference (ICRC 2021)

July 12th - 23rd, 2021

Online - Berlin, Germany

\footnotetext{
*Presenter
} 


\section{Introduction}

The ANTARES neutrino telescope is a water Cherenkov neutrino detector anchored to the seabed at a depth of about 2500 metres, $40 \mathrm{~km}$ offshore from Toulon. It consist of 12 strings composed of 25 storeys, each one with 3 down-facing optical modules (OM). Each OM houses a 10 inch photomultiplier [1]. ANTARES has been operational for more than 13 years and has actively participated in sending alerts for interesting neutrino events to other observatories like the Baikal-GVD experiment.

Baikal-GVD, referred as GVD hereafter, is another water Cherenkov neutrino detector located in the depths of Lake Baikal, Russia. The Baikal-GVD telescope, forward referred as GVD, is organised in clusters. Each cluster holds 8 strings of 36 OMs. Currently 8 of 14 clusters are installed, covering an effective detection volume of $0.40 \mathrm{~km}^{3}$ [2]. From December 2018 to December 2020, GVD received 38 ANTARES alerts and followed up 32 of these as 6 alerts were sent during a period of detector maintenance. After no prompt coincidence was found by GVD (see section 2), further investigation showed some cascades events at an angular distance to the event below $5^{\circ}$ in a time span of \pm 1 day around the alert time for 3 of these alerts. The $4.5^{\circ}$ median angular resolution of GVD for cascades allows for the possibility of these events to be spatially correlated, which makes them of special interest.

ANTARES alerts are based on upgoing track-like events obtained from an online reconstruction. This means that the complete ANTARES data sample, consisting also of downgoing tracks and the cascade channel is still unexplored. For this reason a dedicated offline analysis has been conducted to search for additional events coming from the same direction.

\section{ANTARES alerts}

ANTARES distributes external alerts in case of three classes of events: 1) doublets of neutrinos, that is, detection of at least two neutrino induced muons coming from similar directions of the sky within a predefined time window. 2) In the case of neutrinos with estimated energy $E>1 \mathrm{TeV}$ and direction close to local galaxies (shortened as DIR); 3 ) and for high $(E>7 \mathrm{TeV})$ or very high energy $(E>30 \mathrm{TeV})$ neutrinos, HE trigger and VHE trigger respectively [3].

The skymap for the three ANTARES alerts with coincident cascade events are shown in Figure 1 together with the coincident GVD cascades. Values for the alert time and coordinates are shown in Table 1 together with the trigger type.

GVD's follow-up frame consist of two different searches. The first one looks for events detected by a single cluster in 3 different time windows. First, $+ \pm 500 \mathrm{sec}$ to the alert time (here referred as prompt coincidences), then in \pm 1 hour and, finally, in \pm 1 day. The search is always done around a cone of $5^{\circ}$ around alert position. The second search looks for coincidences on two or more clusters within $6 \mu$ s for the first \pm 10 seconds and, in an extended interval, for the next \pm 1 hour around the trigger. Baikal only found coincident cascades for 3 of the 38 ANTARES alerts inside the $5^{\circ}$ cone and \pm 1 day time window. The coordinates of this cascades can be seen in Figure 1. Values for the detection time of these cascades can be found in table 2. An estimation of the expected background for a single cluster during 24 hours is also given together with the pre-trial p-values reported from GVD. The smallest pre-trial p-value is found for alert A7 being $8.46 \cdot 10^{-4}(3.1 \sigma)$. This is also 

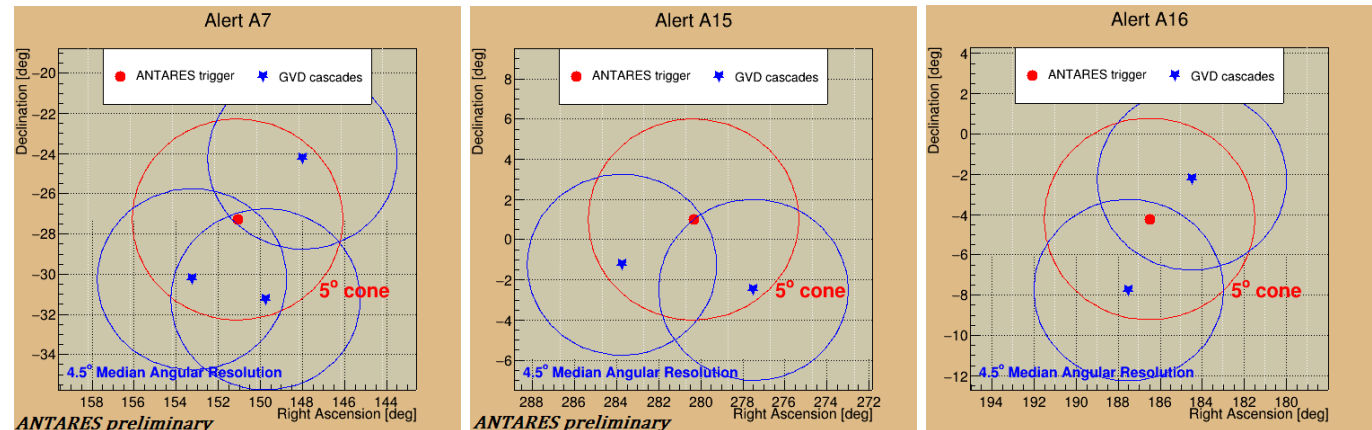

Figure 1: Skymap of the three ANTARES alerts studied in this work. The red circle shows the GVD search cone. The blue circle is the median angular resolution of the GVD detector for cascade events.

\begin{tabular}{|c|c|c|c|c|}
\hline Alert ID & Right Asc. $\left[^{\circ}\right]$ & Declination $\left[{ }^{\circ}\right]$ & MJD & Trigger \\
\hline A7 & 151.1 & -27.3 & 58521.773 & DIR. \\
\hline A15 & 280.4 & 1.0 & 58640.799 & HE \\
\hline A16 & 186.5 & -4.2 & 58645.156 & HE \\
\hline
\end{tabular}

Table 1: Values for the equatorial coordinates, time and trigger type of the three ANTARES alerts with coincident GVD cascades. The time is given in Modified Julian Days, that is, Julian Days respect to the Epoch J2000.0.

\begin{tabular}{|c|c|c|c|c|c|}
\hline Alert ID & $\#$ cas. & $\Delta T_{\text {trigger }}[\mathrm{h}]$ & $\mathrm{Bkg} /$ (clust. day) & $p_{\text {value }}^{\text {pre-trial }}$ & sig. $[\sigma]$ \\
\hline A7 & 3 & $+21.7,-3.2,-23.2$ & 0.090 & $8.46 \cdot 10^{-4}$ & 3.1 \\
\hline A15 & 2 & $+20.3,-0.6$ & 0.108 & $5.2 \cdot 10^{-3}$ & 2.6 \\
\hline A16 & 2 & $-14.8,-18.6$ & 0.090 & $3.6 \cdot 10^{-3}$ & 2.7 \\
\hline
\end{tabular}

Table 2: Values on the GVD coincident cascades arriving times and expected background for a single cluster during a day. The pre-trial p-value is also given and its value in terms of sigmas as reported from GVD. Significance for A7 is computed only for 2 cascades.

a special case as 2 cascades were detected with the same cluster, so the p-value is computed only for cascades detected with different clusters, that is, one cascade has been excluded. Values for the post-trial significance are under investigation and are not presented in this work.

\section{Analysis Method}

For the three alerts for which GVD found coincident cascades (Table 1) the ANTARES collaboration performed a binned analysis to search for additional events from the same sky direction. Binned methods are based on comparing the measured data with the expected background and computing the significance of the excess. For this particular analysis a set of cuts used for event selection, including the size of the region of interest (RoI), is optimised such that, with the resulting background inside the RoI, a single detected event would have a significance of $3 \sigma$.

The main background in the ANTARES neutrino telescopes stems from muons and neutrinos generated in the atmosphere. Muons cannot cross the Earth so for the analysis of events coming 
from above the horizon, the background reach higher levels than for events coming from below. The search time window used on this part of the analysis is 48 hours centred around the time of the alert as distributed by ANTARES. Due to the declination of the three alerts reported in Table 1, they will sometimes be above or below the ANTARES horizon. Table 3 shows for each alert how much time the events coming from that region of the sky are expected to be visible as upgoing or downgoing. For this reason the analysis is divided in two complementary analyses that depends on the zenith direction of the events.

\begin{tabular}{|c|c|c|}
\hline Aler ID & $T_{u p}[\mathrm{~h}]$ & $T_{\text {down }}[\mathrm{h}]$ \\
\hline A7 & 31.7 & 16.3 \\
\hline A15 & 23.7 & 25.3 \\
\hline A16 & 25.2 & 23.8 \\
\hline
\end{tabular}

Table 3: Time in hours that each alert will transverse the sky above or below the ANTARES horizon.

In the same way, different signatures can be distinguished depending on the type of neutrino interaction (charged current or neutral current interaction) and the neutrino flavor. Muon neutrinos interacting through a charged current interaction will manifest themselves with a track-like signature. Neutral current interactions and charged current interactions of electron neutrinos will always manifest themselves as a cascade of many charged particles. Each of this morphologies are parametrised with different variables ([4], Chapter 4.2), thus for each region of the sky the optimisation is divided into the optimisation of the track sample and the optimisation of the cascade sample. The two samples are exclusive since no event belongs to both.

Muon tracks are reconstructed using a multistep procedure that concludes with a maximum likelihood method [5]. This likelihood takes into account the so-called hits. A hit is the digital information on the time and amplitude of a PMT signal.Neutrino-induced muons are selected by applying cuts on the reconstructed zenith angle $\left(\cos \theta_{t r}\right)$, the estimated angular error $\left(\beta_{t r}\right)$, and the parameter that describes the quality of the reconstruction (denoted as $\lambda$ ), [6]. In the present analysis, a cut on the total number of hits, $N_{H i t s}$, is also used. An energy estimator is defined based on the hit charge (recorded by all PMTs used to reconstruct the track) and the length of the muon path in the detector. The selection of higher energy events favor the selection of neutrinos of cosmic origin.

Events not reconstructed as track are considered cascades. They are reconstructed with an algorithm based on a two-step procedure [7]

The background expected inside a selected RoI around a given declination and right ascension is computed from real data. Real data is composed mostly of background so the right ascension is scrambled (blinded) to hide any possible sources. Events are counted inside a band of constant declination and width $2 \times R_{R o I}$, and are later renormalised to the solid angle of the cone. Also, to further increase statistics, data from many days is used, so it has to be also temporally renormalised to the correct time window. Finally, with $N_{\text {cuts }}$ the number of events passing the cuts on the declination band, $N_{b k g}$ is:

$$
N_{\text {bkg }}=N_{\text {cuts }} \times \frac{2 \pi\left(1-\cos R_{\text {RoI }}\right)}{2 \pi\left(\sin \left(\delta_{\text {alert }}+R_{\text {RoI }}\right)-\sin \left(\delta_{\text {alert }}-R_{\text {RoI }}\right)\right)} \times \frac{T_{\text {search }}}{T_{\text {total }}}
$$


As the size of the RoI is a free parameter in the optimisation, for each posible RoI we will find a set of cuts that fixes the expected background below the $3 \sigma$ level. To decide which is the best possible $R_{R o I}$ and cut combination, all the found sets of cuts are compared in terms of resulting acceptance (Acc) to a $E^{-2}$ spectrum, which is expected from Fermi acceleration model for astrophysical sources [8], and the one yielding the highest value is selected.

The acceptance (Acc) measures the sensitivity to a signal with a certain spectrum and relates the number of detected events, $n_{s}$, to the total neutrino flux, $\phi_{0}$, through the following equation:

$$
n_{s}=\mathrm{Acc} \cdot \phi_{0} .
$$

Where $\phi_{0}$ is the normalisation factor of the flux such that $\frac{\mathrm{d} \phi_{v}}{\mathrm{~d} E}=\phi_{0} E^{-2}$ with units of $1 /(\mathrm{GeV}$. $\left.\mathrm{cm}^{2} \cdot s\right)$. Hence, the acceptance has units of neutrinos per unit flux. It is obtained from the effective area of the detector, $A_{e f f}$, which can be defined from the following equation:

$$
n_{s}=\int \frac{\mathrm{d} \phi_{v}(E)}{\mathrm{d} E} \cdot A_{e f f}(E) \mathrm{d} E .
$$

The integral is performed in all of allowed energies, which for the ANTARES neutrino telescope range from $100 \mathrm{GeV}$ to $10^{8} \mathrm{GeV}$. The effective area is a priori unknown and it is computed for each possible cut from Monte Carlo simulation.

This criterion is based on minimising the Model Rejection Factor [9]:

$$
\mathrm{MRF}=\frac{\bar{\mu}_{90 \%}}{n_{s}}
$$

As $\bar{\mu}_{90 \%}$ only depends on the background, which is fixed for all the cuts, the only way of minimising the MRF is to increase $n_{s}$, which is equivalent to finding the highest value for the acceptance as seen in equation (2).

\section{Results}

After completing the optimisation for both interaction channels and sky directions, the data corresponding to the 48 hour time window is explored for additional events. For each event inside the RoI around the alert is searched for a possible track, and if the result is negative, them the cascade condition is tested, and if negative again the events is discarded

No extra signal was detected so limits on the neutrino fluence were computed for a $E^{-2}$ spectrum. Using Poisson statistics, these upper limits correspond to the time integrated flux that would produced on average $n^{90 \%}=2.3$ detected neutrino events. The spectral fluence at the detector can be defined as:

$$
\Phi_{0}^{\mathrm{UL}}\left[\mathrm{GeV} / \mathrm{cm}^{2}\right]=\iint E \frac{\mathrm{d} \phi_{v}}{\mathrm{~d} E} \mathrm{~d} E \mathrm{~d} t=\Delta T \int E \frac{\mathrm{d} \phi_{v}}{\mathrm{~d} E} \mathrm{~d} E .
$$

The spectral fluence is computed for the upgoing and downgoing sky separately so $\Delta T$, which is the search time, correspond to those shown in Table 3. As expected, the cuts for the downgoing sky are much stricter than for the upgoing sky, so the limits are overall a factor 4 worse. This can be seen in Figure 2. The expected error for these values is expected to be around $30 \%$ and $40 \%$ for the upgoing and downgoing sky respectively [10] 

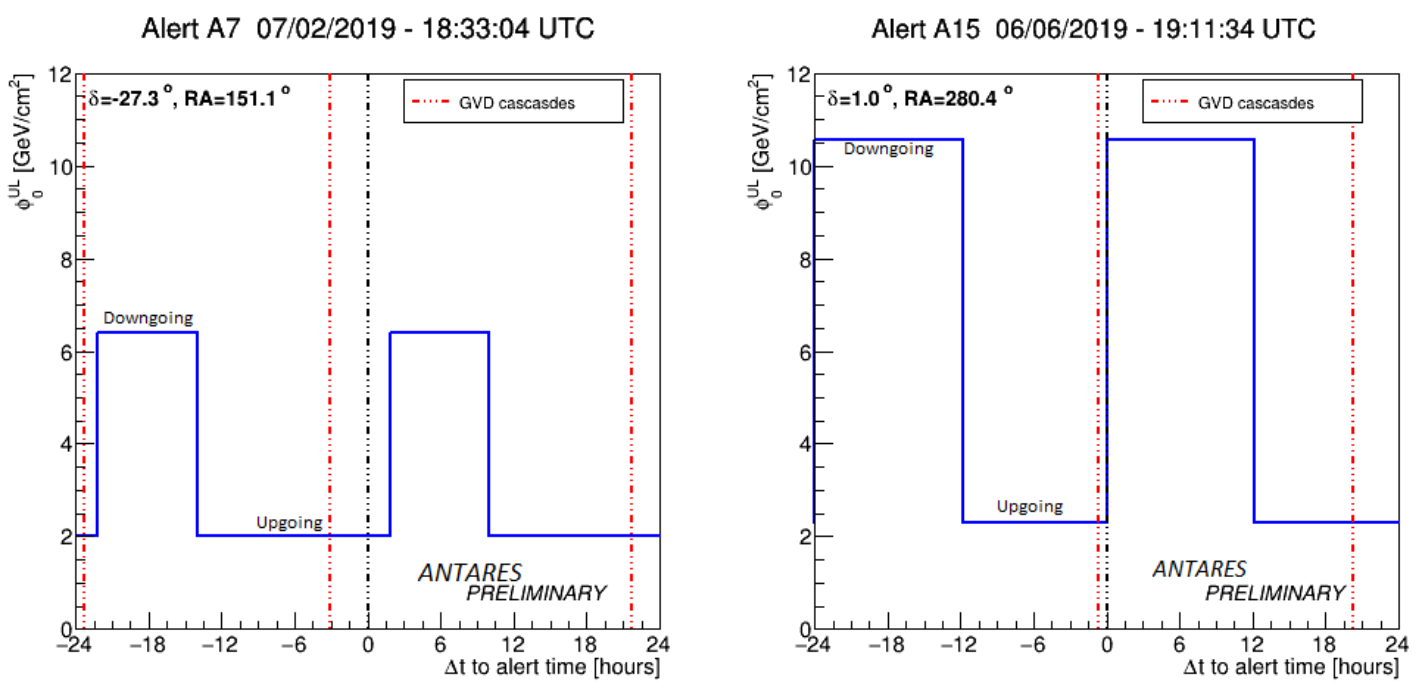

Alert A16 11/06/2019 - 03:45:00 UTC

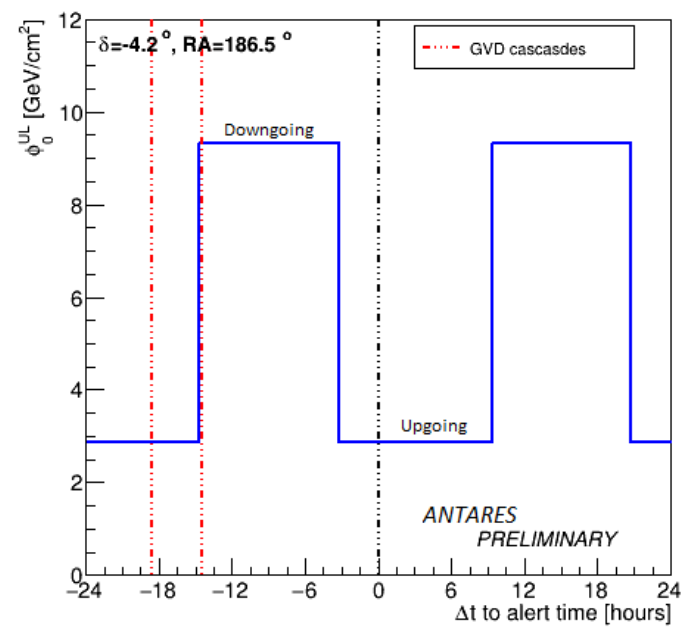

Figure 2: Limits on the neutrino fluence during the time search divided in upgoing and downgoig sky. The red lines represent the detection time of the GVD cascades respect to the alert time.

\section{Conclusion and outlook}

In this analysis we explored the full ANTARES data set around three alerts for which BaikalGVD found time coincident cascades near those three locations on the sky. A binned analysis method was used combined with a set of selection cuts that would ensure a $3 \sigma$ significance in case of extra signal detected. After the unblinding of the events, no extra signal was found near the alerts so limits on the fluence where computed as shown in Figure 2.

As mentioned in the introduction, ANTARES alerts are obtained from an online reconstruction based on an online calibration whereas data is obtained using the same reconstruction algorithm but with a more elaborated detector calibration. This means that the events that triggered the alerts might not manifest again in the offline data. However, that's not the case with the three trigger tracks passing the $3 \sigma$ selection cuts and being reconstructed, at most, $0.5^{\circ}$ from their original direction. 
Obviously they have not been considered, hence quoting the limits on the extra signal.

The significance of Baikal findings is close to the $3 \sigma$ significance as can be seen in table 2 . Considering that the ANTARES tracks are also quite significant when compared to pure background, further efforts are being pursued to compute the joint significance of both findings as the evidence $3 \sigma$ threshold could be surpassed, specially for alert A7.

\section{Acknowledgments}

This work was supported by the Generalitat Valenciana, Spain. Grant CIDEGENT 2018/034. This work was supported bt RFBR grants 2002-00400 and 19-29-11029.

\section{References}

[1] M. Ageron, Aguilar et al., ANTARES: the first undersea neutrino telescope, Nuclear Instruments and Methods in Physics Research Section A: Accelerators, Spectrometers, Detectors and Associated Equipment 656 (2011) 11.

[2] A. Avrorin et al., The Baikal-GVD neutrino telescope: First results of multi-messenger studies, arXiv preprint arXiv:1908.05450 (2019) .

[3] M. Ageron et al., The ANTARES telescope neutrino alert system, Astroparticle Physics $\mathbf{3 5}$ (2012) 530 .

[4] G. Illuminati, Searches for cosmic neutrino sources with ANTARES, KM3NeT and IceCube and time calibration of ANTARES, .

[5] Adrián-Martínez et al., Search for cosmic neutrino point sources with four years of data from the antares telescope, The Astrophysical Journal 760 (2012) 53.

[6] Adrián-Martínez et al., Searches for point-like and extended neutrino sources close to the galactic center using the antares neutrino telescope, The Astrophysical journal letters $\mathbf{7 8 6}$ (2014) L5.

[7] A. Albert, André et al., An algorithm for the reconstruction of high-energy neutrino-induced particle showers and its application to the ANTARES neutrino telescope, The European Physical Journal C 77 (2017) 1.

[8] E. Fermi, On the origin of the cosmic radiation, Physical review 75 (1949) 1169.

[9] G.C. Hill and K. Rawlins, Unbiased cut selection for optimal upper limits in neutrino detectors: the model rejection potential technique, Astroparticle physics 19 (2003) 393.

[10] A. Albert, André et al., Search for neutrino counterparts of gravitational-wave events detected by LIGO and Virgo during run o2 with the ANTARES telescope, The European Physical Journal C 80 (2020) 1. 


\section{Full Authors List: ANTARES Collaboration}

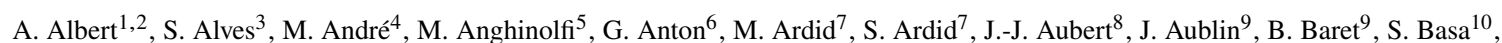

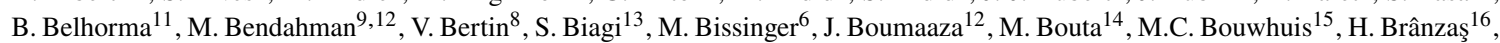
R. Bruijn ${ }^{15,17}$, J. Brunner ${ }^{8}$, J. Busto ${ }^{8}$, B. Caiff ${ }^{5}$, A. Capone ${ }^{18,19}$, L. Caramete ${ }^{16}$, J. Carr ${ }^{8}$, V. Carretero ${ }^{3}$, S. Celli ${ }^{18,19}$, M. Chabab ${ }^{20}$, T. N. Chau ${ }^{9}$, R. Cherkaoui El Moursli ${ }^{12}$, T. Chiarusi ${ }^{21}$, M. Circella $^{22}$, A. Coleiro ${ }^{9}$, M. Colomer-Molla ${ }^{9,3}$, R. Coniglione $^{13}$, P. Coyle $^{8}$, A. Creusot $^{9}$, A. F. Díaz ${ }^{23}$, G. de Wasseige ${ }^{9}$, A. Deschamps ${ }^{24}$, C. Distefano ${ }^{13}$, I. Di Palma ${ }^{18,19}$, A. Domi ${ }^{15,17}$, C. Donzaud ${ }^{9,25}$, D. Dornic ${ }^{8}$, D. Drouhin ${ }^{1,2}$, T. Eberl ${ }^{6}$, T. van Eeden ${ }^{15}$, D. van Eijk ${ }^{15}$, N. El Khayati ${ }^{12}$, A. Enzenhöfer ${ }^{8}$, P. Fermani ${ }^{18,19}$, G. Ferrara $^{13}$, F. Filippini ${ }^{21,26}$, L.A. Fusco ${ }^{8}$, Y. Gatelet ${ }^{9}$, P. Gay ${ }^{27,9}$, H. Glotin $^{28}$, R. Gozzini ${ }^{3}$, R. Gracia Ruiz ${ }^{15}$, K. Graf ${ }^{6}$, C. Guidi ${ }^{5,29}$, S. Hallmann ${ }^{6}$, H. van Haren ${ }^{30}$, A.J. Heijboer ${ }^{15}$, Y. Hello ${ }^{24}$, J.J. Hernández-Rey ${ }^{3}$, J. Höß1 ${ }^{6}$, J. Hofestädt ${ }^{6}$, F. Huang ${ }^{8}$, G. Illuminati ${ }^{9}, 21,26$, C.W James ${ }^{31}$, B. Jisse-Jung ${ }^{15}$, M. de Jong ${ }^{15,32}$, P. de Jong ${ }^{15}$, M. Kadler ${ }^{33}$, O. Kalekin ${ }^{6}$, U. Katz ${ }^{6}$, N.R. Khan-Chowdhury ${ }^{3}$, A. Kouchner ${ }^{9}$, I. Kreykenbohm ${ }^{34}$, V. Kulikovskiy ${ }^{5,36}$, R. Lahmann ${ }^{6}$, R. Le Breton ${ }^{9}$, D. Lefèvre ${ }^{35}$, E. Leonora ${ }^{36}$, G. Levi ${ }^{21,26}$, M. Lincetto ${ }^{8}$, D. Lopez-Coto ${ }^{37}$, S. Loucatos ${ }^{38,9}$, L. Maderer ${ }^{9}$, J. Manczak ${ }^{3}$, M. Marcelin ${ }^{10}$, A. Margiotta ${ }^{21,26}$, A. Marinelli ${ }^{39}$, J.A. Martínez-Mora ${ }^{7}$,

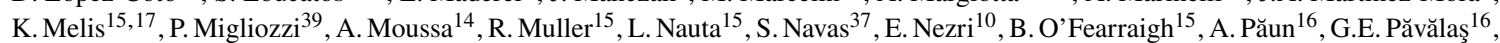
C. Pellegrino ${ }^{21,40,41}$, M. Perrin-Terrin ${ }^{8}$, V. Pestel ${ }^{15}$, P. Piattelli ${ }^{13}$, C. Pieterse ${ }^{3}$, C. Poirè ${ }^{7}$, V. Popa ${ }^{16}$, T. Pradier ${ }^{1}$, N. Randazzo ${ }^{36}$, S. Reck ${ }^{6}$, G. Riccobene ${ }^{13}$, A. Romanov ${ }^{5,29}$, A. Sánchez-Losa ${ }^{3,22}$, F. Salesa Greus ${ }^{3}$, D. F. E. Samtleben ${ }^{15,32}$, M. Sanguineti ${ }^{5,29}$, P. Sapienza ${ }^{13}$, J. Schnabel ${ }^{6}$, J. Schumann ${ }^{6}$, F. Schüssler ${ }^{38}$, M. Spurio ${ }^{21,26}$, Th. Stolarczyk ${ }^{38}$, M. Taiuti ${ }^{5,29}$, Y. Tayalati ${ }^{12}$, S.J. Tingay ${ }^{31}$, B. Vallage ${ }^{38,9}$, V. Van Elewyck ${ }^{9,41}$, F. Versari ${ }^{21,26,9}$, S. Viola ${ }^{13}$, D. Vivolo ${ }^{39,43}$, J. Wilms ${ }^{34}$, S. Zavatarelli ${ }^{5}$, A. Zegarelli ${ }^{18,19}$, J.D. Zornoza ${ }^{3}$, and J. Zúñiga ${ }^{3}$

${ }^{1}$ Université de Strasbourg, CNRS, IPHC UMR 7178, F-67000 Strasbourg, France. ${ }^{2}$ Université de Haute Alsace, F-68100 Mulhouse, France. ${ }^{3}$ IFIC - Instituto de Física Corpuscular (CSIC - Universitat de València) c/ Catedrático José Beltrán, 2 E-46980 Paterna, Valencia, Spain. ${ }^{4}$ Technical University of Catalonia, Laboratory of Applied Bioacoustics, Rambla Exposició, 08800 Vilanova i la Geltrú, Barcelona, Spain. ${ }^{5}$ INFN - Sezione di Genova, Via Dodecaneso 33, 16146 Genova, Italy. ${ }^{6}$ Friedrich-Alexander-Universität Erlangen-Nürnberg, Erlangen Centre for Astroparticle Physics, Erwin-Rommel-Str. 1, 91058 Erlangen, Germany. ${ }^{7}$ Institut d'Investigació per a la Gestió Integrada de les Zones Costaneres (IGIC) - Universitat Politècnica de València. C/ Paranimf 1, 46730 Gandia, Spain. ${ }^{8}$ Aix Marseille Univ, CNRS/IN2P3, CPPM, Marseille, France. ${ }^{9}$ Université de Paris, CNRS, Astroparticule et Cosmologie, F-75013 Paris, France. ${ }^{10}$ Aix Marseille Univ, CNRS, CNES, LAM, Marseille, France. ${ }^{11}$ National Center for Energy Sciences and Nuclear Techniques, B.P.1382, R. P.10001 Rabat, Morocco. ${ }^{12}$ University Mohammed V in Rabat, Faculty of Sciences, 4 av. Ibn Battouta, B.P. 1014, R.P. 10000 Rabat, Morocco. ${ }^{13}$ INFN - Laboratori Nazionali del Sud (LNS), Via S. Sofia 62, 95123 Catania, Italy. ${ }^{14}$ University Mohammed I, Laboratory of Physics of Matter and Radiations, B.P.717, Oujda 6000, Morocco. ${ }^{15}$ Nikhef, Science Park, Amsterdam, The Netherlands. ${ }^{16}$ Institute of Space Science, RO-077125 Bucharest, Măgurele, Romania. ${ }^{17}$ Universiteit van Amsterdam, Instituut voor Hoge-Energie Fysica, Science Park 105, 1098 XG Amsterdam, The Netherlands. ${ }^{18}$ INFN - Sezione di Roma, P.le Aldo Moro 2, 00185 Roma, Italy. ${ }^{19}$ Dipartimento di Fisica dell'Università La Sapienza, P.le Aldo Moro 2, 00185 Roma, Italy. ${ }^{20}$ LPHEA, Faculty of Science - Semlali, Cadi Ayyad University, P.O.B. 2390, Marrakech, Morocco. ${ }^{21}$ INFN - Sezione di Bologna, Viale Berti-Pichat 6/2, 40127 Bologna, Italy. ${ }^{22}$ INFN - Sezione di Bari, Via E. Orabona 4, 70126 Bari, Italy. ${ }^{23}$ Department of Computer Architecture and Technology/CITIC, University of Granada, 18071 Granada, Spain. ${ }^{24}$ Géoazur, UCA, CNRS, IRD, Observatoire de la Côte d'Azur, Sophia Antipolis, France. ${ }^{25}$ Université Paris-Sud, 91405 Orsay Cedex, France. ${ }^{26}$ Dipartimento di Fisica e Astronomia dell'Università, Viale Berti Pichat 6/2, 40127 Bologna, Italy. ${ }^{27}$ Laboratoire de Physique Corpusculaire, Clermont Université, Université Blaise Pascal, CNRS/IN2P3, BP 10448, F-63000 Clermont-Ferrand, France. ${ }^{28}$ LIS, UMR Université de Toulon, Aix Marseille Université, CNRS, 83041 Toulon, France. ${ }^{29}$ Dipartimento di Fisica dell'Università, Via Dodecaneso 33, 16146 Genova, Italy. ${ }^{30}$ Royal Netherlands Institute for Sea Research (NIOZ), Landsdiep 4, 1797 SZ 't Horntje (Texel), the Netherlands. ${ }^{31}$ International Centre for Radio Astronomy Research - Curtin University, Bentley, WA 6102, Australia. ${ }^{32}$ Huygens-Kamerlingh Onnes Laboratorium, Universiteit Leiden, The Netherlands. ${ }^{33}$ Institut für Theoretische Physik und Astrophysik, Universität Würzburg, Emil-Fischer Str. 31, 97074 Würzburg, Germany. ${ }^{34}$ Dr. Remeis-Sternwarte and ECAP, Friedrich-Alexander-Universität Erlangen-Nürnberg, Sternwartstr. 7, 96049 Bamberg, Germany. ${ }^{35}$ Mediterranean Institute of Oceanography (MIO), Aix-Marseille University, 13288, Marseille, Cedex 9, France; Université du Sud Toulon-Var, CNRS-INSU/IRD UM 110, 83957, La Garde Cedex, France. ${ }^{36}$ INFN - Sezione di Catania, Via S. Sofia 64, 95123 Catania, Italy. ${ }^{37}$ Dpto. de Física Teórica y del Cosmos \& C.A.F.P.E., University of Granada, 18071 Granada, Spain. ${ }^{38}$ IRFU, CEA, Université Paris-Saclay, F-91191 Gif-sur-Yvette, France. ${ }^{39}$ INFN - Sezione di Napoli, Via Cintia 80126 Napoli, Italy. ${ }^{40}$ Museo Storico della Fisica e Centro Studi e Ricerche Enrico Fermi, Piazza del Viminale 1, 00184, Roma. ${ }^{41}$ INFN - CNAF, Viale C. Berti Pichat 6/2, 40127, Bologna. ${ }^{42}$ Institut Universitaire de France, 75005 Paris, France. ${ }^{43}$ Dipartimento di Fisica dell'Università Federico II di Napoli, Via Cintia 80126, Napoli, Italy. 


\section{Full Authors List: Baikal-GVD Collaboration}

V.A. Allakhverdyan ${ }^{1}$ A.D. Avrorin ${ }^{2}$, A.V. Avrorin ${ }^{2}$, V.M. Aynutdinov ${ }^{2}$, R. Bannasch ${ }^{3}$, Z. Bardačová ${ }^{4}$, I.A. Belolaptikov ${ }^{1}$, I.V. Borina ${ }^{1}$, V.B. Brudanin ${ }^{1}{ }^{*}$, N.M. Budnev ${ }^{5}$, V.Y. Dik ${ }^{1}$, G.V. Domogatsky ${ }^{2}$, A.A. Doroshenko ${ }^{2}$, R. Dvornický ${ }^{1,4}$, A.N. Dyachok ${ }^{5}$, Zh.-A.M. Dzhilkibaev ${ }^{2}$, E. Eckerová, ${ }^{4}$, T.V. Elzhov $^{1}$, L. Fajt $^{6}$, S.V. Fialkovski ${ }^{7}{ }^{*}$, A.R. Gafarov ${ }^{5}$, K.V. Golubkov ${ }^{2}$, N.S. Gorshkov ${ }^{1}$, T.I. Gress ${ }^{5}$, M.S. Katulin ${ }^{1}$, K.G. Kebkal ${ }^{3}$, O.G. Kebkal ${ }^{3}$, E.V. Khramov ${ }^{1}$, M.M. Kolbin ${ }^{1}$, K.V. Konischev ${ }^{1}$, K.A. Kopański ${ }^{8}$, A.V. Korobchenko ${ }^{1}$, A.P. Koshechkin ${ }^{2}$, V.A. Kozhin ${ }^{9}$, M.V. Kruglov' ${ }^{1}$, M.K. Kryukov², V.F. Kulepov ${ }^{7}$, Pa. Malecki ${ }^{8}$, Y.M. Malyshkin ${ }^{1}$, M.B. Milenin ${ }^{2}$, R.R. Mirgazov ${ }^{5}$, D.V. Naumov ${ }^{1}$, V. Nazari ${ }^{1}$, W. $\mathrm{Noga}^{8}$, D.P. Petukhov ${ }^{2}$, E.N. Pliskovsky ${ }^{1}$, M.I. Rozanov ${ }^{10}$, V.D. Rushay ${ }^{1}$, E.V. Ryabov ${ }^{5}$, G.B. Safronov ${ }^{2}$, B.A. Shaybonov ${ }^{1}$, M.D. Shelepov ${ }^{2}$, F. Šimkovic ${ }^{1,4,6}$, A.E. Sirenko ${ }^{1}$, A.V. Skurikhinn ${ }^{9}$, A.G. Solovjev ${ }^{1}$, M.N. Sorokovikov ${ }^{1}$, I. Štekl ${ }^{6}$, A.P. Stromakov ${ }^{2}$, E.O. Sushenok ${ }^{1}$, O.V. Suvorova ${ }^{2}$, V.A. Tabolenko ${ }^{5}$, B.A. Tarashansky ${ }^{5}$, Y.V. Yablokova ${ }^{1}$, S.A. Yakovlev ${ }^{3}$, and D.N. Zaborov ${ }^{2}$

${ }^{1}$ Joint Institute for Nuclear Research, Dubna, Russia. ${ }^{2}$ Institute for Nuclear Research, Russian Academy of Sciences, Moscow, Russia. ${ }^{3}$ EvoLogics GmbH, Berlin, Germany. ${ }^{4}$ Comenius University, Bratislava, Slovakia. ${ }^{5}$ Irkutsk State University, Irkutsk, Russia. ${ }^{6}$ Czech Technical University in Prague, Prague, Czech Republic. ${ }^{7}$ Nizhny Novgorod State Technical University, Nizhny Novgorod, Russia. ${ }^{8}$ Institute of Nuclear Physics of Polish Academy of Sciences (IFJ PAN), Kraków, Poland. ${ }^{9}$ Skobeltsyn Institute of Nuclear Physics, Moscow State University, Moscow, Russia. ${ }^{10}$ St. Petersburg State Marine Technical University, St.Petersburg, Russia. *Deceased. 